\title{
A randomized, multicenter, phase II/III study to determine the optimal dose and to evaluate the efficacy and safety of pegteograstim (GCPGC) on chemotherapy-induced neutropenia compared to pegfilgrastim in breast cancer patients: KCSG PC10-09
}

\author{
Ki Hyeong Lee ${ }^{1}$ Ji-Yeon Kim ${ }^{2}$ Moon Hee Lee ${ }^{3}$ Hye Sook Han ${ }^{1} \cdot J_{0 o}$ Han Lim ${ }^{3}$ • \\ Keon Uk Park ${ }^{4}$ - In Hae Park ${ }^{5}$ Eun Kyung Cho ${ }^{6}$ - So Young Yoon ${ }^{7}$. Jee Hyun Kim ${ }^{8}$. \\ In Sil Choi ${ }^{9}$. Jae Hoo Park ${ }^{10}$ • Young Jin Choi ${ }^{11}$ • Hee-Jun Kim ${ }^{12}$ • Kyung Hae Jung ${ }^{13}$. \\ Si-Young Kim ${ }^{14}$ • Do-Youn $\mathrm{Oh}^{2,15} \cdot$ Seock-Ah Im $^{2,15}$
}

Received: 10 June 2015 / Accepted: 21 September 2015 / Published online: 1 October 2015

(C) The Author(s) 2015. This article is published with open access at Springerlink.com

\begin{abstract}
Purpose Pegylated granulocyte-colony-stimulating factor (G$\mathrm{CSF}$ ) is frequently used to prevent febrile neutropenia (FN) in patients undergoing chemotherapy with a high risk of myelosuppression. This phase II/III study was conducted to determine the adequate dose of pegteograstim, a new formulation of pegylated G-CSF, and to evaluate the efficacy and safety of pegteograstim compared to pegfilgrastim.
\end{abstract}

Do-Youn Oh

ohdoyoun@snu.ac.kr

Seock-Ah Im

moisa@snu.ac.kr

1 Division of Medical Oncology, Department of Internal Medicine, Chungbuk National University Hospital, CheongJu, South Korea

2 Division of Hematology and Oncology, Department of Internal Medicine, Seoul National University Hospital, Cancer Research Institute, Seoul National University College of Medicine, Seoul, South Korea

3 Division of Hemato-oncology, Department of Internal Medicine, Inha University Hospital, Incheon, South Korea

4 Division of Hematology/Oncology, Department of Internal Medicine, Keimyung University Dongsan Hospital, Daegu, South Korea

5 Center for Breast Cancer, Hospital of the National Cancer Center, National Cancer Center, Goyang, South Korea

6 Division of Hematology and Oncology, Department of Internal Medicine, Gachon University Gil Medical Center, Incheon, South Korea
Methods In the phase II part, 60 breast cancer patients who were undergoing DA (docetaxel and doxorubicin) or TAC (docetaxel, doxorubicin, and cyclophosphamide) chemotherapy were randomly selected to receive a single subcutaneous injection of 3.6 or $6.0 \mathrm{mg}$ pegteograstim on day 2 of each chemotherapy cycle. The phase III part was seamlessly started to compare the dose of pegteograstim at selected in phase II with $6.0 \mathrm{mg}$ pegfilgrastim in 117 breast cancer patients. The

7 Division of Hematooncology, Department of Internal Medicine, Konkuk University Medical Center, Konkuk University School of Medicine, Seoul, South Korea

8 Division of Hematology and Medical Oncology, Department of Internal Medicine, Seoul National University Bundang Hospital, Seongnam, South Korea

9 Division of Hematology/Oncology, Department of Internal Medicine, SMG-SNU Boramae Medical Center, Seoul, South Korea

10 Division of Hematology and Oncology, Department of Internal Medicine, Ulsan University Hospital, Ulsan, South Korea

11 Division of Hemato-oncology, Department of Internal Medicine, Pusan National University Hospital, Busan, South Korea

12 Division of Hematology/Medical Oncology, Department of Internal Medicine, Chung-Ang University Hospital, Seoul, South Korea

13 Department of Oncology, Asan Medical Center, University of Ulsan College of Medicine, Seoul, South Korea

14 Division of Medical Oncology/Hematology, Department of Internal Medicine, Kyung Hee University Hospital, Seoul, South Korea

15 Department of Internal Medicine, Seoul National University Hospital, Cancer Research Institute, Seoul National University College of Medicine, 101 Daehak-ro, Jongno-gu, Seoul 110-744, South Korea 
primary endpoint of both the phase II and III parts was the duration of grade 4 neutropenia in the chemotherapy cycle 1 . Results The mean duration of grade 4 neutropenia for the $3.6 \mathrm{mg}$ pegteograstim $(n=33)$ was similar to that for the $6.0 \mathrm{mg}$ pegteograstim $(n=26)(1.97 \pm 1.79$ days vs. $1.54 \pm 0.95$ days, $p=0.33$ ). The $6.0 \mathrm{mg}$ pegteograstim was selected to be compared with the $6.0 \mathrm{mg}$ pegfilgrastim in the phase III part. In the phase III part, the primary analysis revealed that the efficacy of pegteograstim $(n=56)$ was noninferior to that of pegfilgrastim $(n=59)$ [duration of grade 4 neutropenia, $1.64 \pm 1.18$ days vs. $1.80 \pm 1.05$ days; difference, $-0.15 \pm 1.11(p=0.36,97.5 \%$ confidence intervals $=0.57$ and $0.26)]$. The time to the absolute neutrophil count (ANC) recovery of pegteograstim $(\geq 2000 / \mu \mathrm{L})$ was significantly shorter than that of pegfilgrastim $(8.85 \pm 1.45$ days vs. $9.83 \pm 1.20$ days, $p<0.0001)$. Other secondary endpoints showed no significant difference between the two groups. The safety profiles of the two groups did not differ significantly.

Conclusions Pegteograstim was shown to be as effective as pegfilgrastim in the reduction of chemotherapy-induced neutropenia in the breast cancer patients who were undergoing chemotherapy with a high risk of myelosuppression.

Keywords Chemotherapy-induced neutropenia $\cdot$ Breast cancer $\cdot$ Pegylated G-CSF · Pegteograstim $\cdot$ Prophylaxis

\section{Introduction}

Myelosuppression is the most common form of dose-limiting toxicity of conventional anticancer chemotherapies, which restricts their application. Chemotherapy-induced febrile neutropenia (FN) is especially life threatening in some patients, requiring hospitalization, intravenous (IV) antibiotic therapy, and dose reduction in subsequent cycles, which compromise the efficacy of chemotherapy. Thus, recent guidelines recommend primary or secondary prophylaxis with granulocytecolony-stimulating factors (G-CSFs) before using FN highrisk chemotherapy regimens or before subsequent cycles after the development of FN in the previous cycle [1].

Whereas filgrastim has a plasma half-life of $110 \mathrm{~min}$ and requires daily injection [2], pegfilgrastim (Neulasta ${ }^{\circledR}$, Amgen, Thousand Oaks, CA, USA), a pegylated form of filgrastim, has an increased half-life (about $33.2 \mathrm{~h}$ ) and duration of action (about 8.3 days) [3, 4]. Generally, pegfilgrastim is administered monthly, and conventional G-CSF is dosed daily, which implies the comparable efficacy of pegfilgrastim with improved convenience and safety $[5,6]$. Several studies showed that pegfilgrastim is associated with a lower risk of hospitalization of cancer patients than filgrastim and cost-effective. [7-9].
Pegteograstim is a novel recombinant human G-CSF conjugated with methoxy-maleimide-polyethylene glycol, which was developed by Green Cross Corporation in Korea. As it bears a PEG anchored to a unique site in the structurally flexible CD-loop region of G-CSF, which is located far from receptor binding sites, it has higher affinity to the G-CSF receptor and correspondingly higher in vitro biological activity than pegfilgrastim (data not shown).

In the previous phase I study, the PK-PD characteristics of $30-100 \mu \mathrm{g} / \mathrm{kg}$ of pegteograstim were comparable to those of $100 \mu \mathrm{g} / \mathrm{kg}$ of pegfilgrastim, and $100 \mu \mathrm{g} / \mathrm{kg}$ of pegteograstim showed a $20 \%$ greater increase in their absolute neutrophil count (ANC) and $\mathrm{CD} 34^{+}$cell counts than pegfilgrastim. Furthermore, $30-100 \mu \mathrm{g} / \mathrm{kg}$ of pegteograstim was well tolerated in healthy Korean males [10].

Based on the phase I study, we conducted a multicenter, randomized, seamless phase II/III study to demonstrate the non-inferiority of pegteograstim to pegfilgrastim in breast cancer patients, in terms of the duration of grade 4 neutropenia (ANC $<500 / \mu \mathrm{L}$ ) during the first chemotherapy cycle. Before proceeding to the phase III part, we explored the optimal dose of pegteograstim in the phase II part by comparing different doses of pegteograstim.

\section{Materials and methods}

\section{Patients}

Female patients were eligible for inclusion in this study if they had breast cancer and planned to undergo DA $\left(75 \mathrm{mg} / \mathrm{m}^{2}\right.$ docetaxel plus $50 \mathrm{mg} / \mathrm{m}^{2}$ doxorubicin on day 1 every 3 weeks) chemotherapy or TAC $\left(75 \mathrm{mg} / \mathrm{m}^{2}\right.$ docetaxel plus $50 \mathrm{mg} / \mathrm{m}^{2}$ doxorubicin plus $500 \mathrm{mg} / \mathrm{m}^{2}$ cyclophosphamide on day 1 every 3 weeks) chemotherapy. The other eligibility criteria included an age of at least 18 years, body weight $\geq 45 \mathrm{~kg}$, Eastern Cooperative Oncology Group (ECOG) performance status $\leq 2$, ANC $\geq 1500 / \mu \mathrm{L}$, platelet count $\geq 100000 / \mu \mathrm{L}$, and life expectancy $\geq 3$ months. The exclusion criteria were exposure to antibiotics within $72 \mathrm{~h}$ before the chemotherapy; moderate to severe heart failure according to the New York Heart Association functional classification III-IV or clinically significant cardiac dysfunction; a history of bone marrow or stem cell transplantation; exposure to pegfilgrastim, filgrastim, sargramostim (GM-CSF), or lenograstim within 4 weeks of the patient's consent to participate in this study; pregnancy or breast feeding female patients; cystitis or urinary outlet obstruction; and major surgery unrelated to anticancer treatment within 4 weeks of the patient registration. All the female patients provided their written informed consent to participate in this study. 


\section{Study design}

This study is a randomized, multicenter, seamless phase II (single-blinded)/III (double-blinded) study (Fig. 1). In the phase II part, the female patients were randomized (1:1) to receive either a subcutaneous injection of 3.6 or $6.0 \mathrm{mg}$ pegteograstim in a single-blinded manner on day 2 of each chemotherapy cycle. Although up to four cycles of chemotherapy were planned, up to six cycles of additional treatments were allowed at the discretion of the investigators. In addition, follow-up was conducted 12 weeks after the completion of the study treatment to determine the anti-drug antibody formation. The phase III part was designed to test the non-inferiority of pegteograstim to pegfilgrastim. The female patients were randomized at 1:1 in a double-blinded manner to receive either the study drug $(6.0 \mathrm{mg}$ pegteograstim, the dose selected in phase II) or the comparator (6.0 mg pegfilgrastim). The randomization was stratified according to the type of chemotherapy (DA versus TAC), and the patients received the study medication on day 2 of each chemotherapy cycle, for up to six cycles.

This study was conducted in full accordance with the guidelines for Good Clinical Practice and the Declaration of Helsinki. The Korean Cancer Study Group (KCSG) Protocol Review Committee approved the study protocol, and the approval of the Institutional Ethics Committees was obtained before the start of the patient accrual at each institution. This trial has been registered at ClinicalTrials.gov as NCT01328938.

\section{Phase II}

The female patients were intravenously administered four to six cycles of DA or TAC chemotherapy. They were randomly assigned to group I $(3.6 \mathrm{mg})$ or group II $(6.0 \mathrm{mg})$ with stratification by institution and type of chemotherapy (DA vs. TAC). Each female patient received a single subcutaneous injection of pegteograstim at either dose on day $2(24 \mathrm{~h}$ after the chemotherapy) of each cycle in a single-blinded manner. After the optimal or appropriate dose of pegteograstim was selected, the study seamlessly proceeded to phase III.

\section{Phase III}

The female patients were administered the same chemotherapy regimen and schedules as in phase II but received the dose of pegteograstim at selected in phase II or the comparator ( $6.0 \mathrm{mg}$ of pegfilgrastim) in a double-blinded manner. The patients who required therapeutic G-CSF administration at the discretion of the investigators were excluded from the efficacy analyses. Prophylactic antibiotic therapy was not allowed during cycle 1 , but allowed from cycle 2 , based on the judgment of the investigators for those who suffered persistent ( $>7$ days) grade 4 neutropenia in cycle 1 . Corticosteroid use was prohibited, except for anti-emetic purposes.

\section{Outcome measurements}

The primary endpoint in both the phase II and III parts was the duration (in days) of grade 4 neutropenia during the first chemotherapy cycle in both phases. Grade 4 neutropenia was defined as ANC $<500 / \mu \mathrm{L}$. The secondary endpoints in phase II were the time (in days) to the recovery to ANC $\geq 2000 / \mu \mathrm{L}$ after the nadir during cycle 1 , the incidence of $\mathrm{FN}$, the safety profile, and the formation of an anti-drug antibody against the administered study drug. FN was defined as a condition with an $\mathrm{ANC}<500 / \mu \mathrm{L}$, and fever, as a condition with a single oral temperature of $\geq 38.3{ }^{\circ} \mathrm{C}$ or two or more instances of $\geq 38.0^{\circ} \mathrm{C}$ each taken within 1-24 $\mathrm{h}$ after the initial measurement. In phase III, the secondary endpoints were the time (in days) to the recovery to ANC $\geq 2000 / \mu \mathrm{L}$ after the ANC nadir during cycle 1, the incidence of FN, the ANC nadir during cycle 1, the incidence of persistent grade 4 neutropenia over 3 days or longer, the ANC value on day 7 during all the chemotherapy cycles, the number of days of hospitalization due to $\mathrm{FN}$ after cycle 2, the number of cases of IV antimicrobial usage due to $\mathrm{FN}$, and incidence of delay or dose reduction of chemotherapy.

\section{Statistical analyses}

To determine the optimal dose of pegteograstim between 3.6 and $6.0 \mathrm{mg}$ at the one-sided $\alpha$ level of 0.1 and the power of

Fig. 1 Overall scheme of study

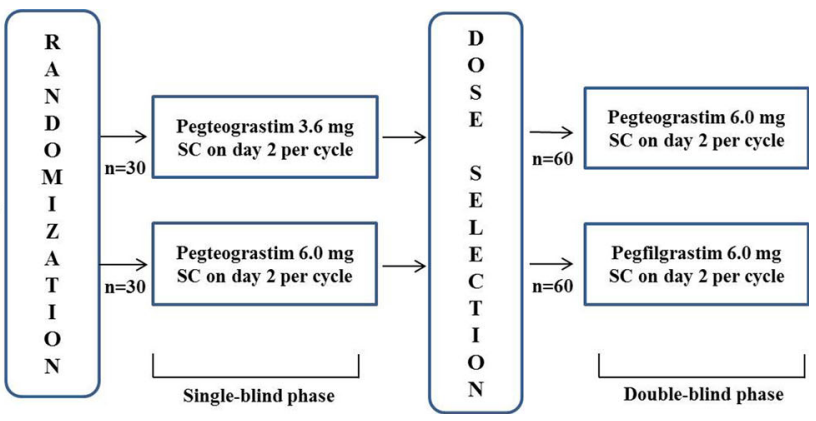


$80 \%$, phase II required 30 patients in each group. In phase III, we estimated the need for 60 patients in each group to demonstrate the non-inferiority of pegteograstim to pegfilgrastim at the one-sided $\alpha$ level of 0.025 and the power of $90 \%$. Noninferiority was concluded if the upper limit of the $97.5 \%$ onesided confidence interval of the inter-group difference in the duration of grade 4 neutropenia did not exceed the limit of 1.0, which was in accordance with the non-inferiority margin.

The primary endpoint was analyzed using the modified intention-to-treat (ITT) set, which is defined as the patients who received the study drug at least once and were evaluable for the efficacy after the first cycle. Safety analyses were conducted in the safety analysis (SA) set, which included any subject who received the study drug at least once.

\section{Results}

\section{Patients}

Between October 2010 and January 2012, 60 female patients were enrolled in the phase II part of this study (Table 1). Thirty-four patients were randomized to the $3.6 \mathrm{mg}$ pegteograstim group, and 26, to the $6.0 \mathrm{mg}$ group. Two patients in the $3.6 \mathrm{mg}$ group were withdrawn without completing cycle 1 due to their inadequate baseline ANC (ANC $<1500$ / $\mu \mathrm{L})$. Following the analysis to determine the optimal dose of pegteograstim after phase II, 117 female patients were recruited from February 2012 to May 2013 for the phase III part (Fig. 2). Fifty-eight patients were randomized to the pegteograstim group, and 59, to the pegfilgrastim group. One patient in the pegteograstim group who did not receive the study drug due to violation of the inclusion criteria was excluded from the safety analyses. One patient randomized to the pegteograstim group received the study drug but was excluded from the analyses because she underwent the G-CSF treatment during FN in cycle 1, and 115 female patients (56 in the pegteograstim group and 59 in the pegfilgrastim group) were assessed for efficacy. The mean age of the subjects in both groups was 49 years.

Overall, $18.2 \%(32 / 176)$ of the patients had previously undergone chemotherapy, and $13.1 \%(23 / 176)$ had undergone adjuvant radiotherapy, with no imbalance between the groups. Of the patients, $73.3 \%(129 / 176)$ underwent adjuvant/neoadjuvant chemotherapy as the study protocol regimen and $26.7 \%$ (47/176) underwent first-line palliative chemotherapy as the study protocol regimen, without imbalance between the groups. Sub-analysis was conducted to compare the FN incidence by the treatment intents, palliative vs. adjuvant/neoadjuvant. FN rates of palliative treatment were higher

Table 1 Demographic and baseline characteristics

\begin{tabular}{|c|c|c|c|c|c|c|c|c|c|c|}
\hline & \multicolumn{5}{|c|}{ Phase II } & \multicolumn{5}{|c|}{ Phase III } \\
\hline & \multicolumn{2}{|c|}{$\begin{array}{l}\text { Pegteograstim } 3.6 \mathrm{mg} \\
(N=34)\end{array}$} & \multicolumn{2}{|c|}{$\begin{array}{l}\text { Pegteograstim } 6.0 \mathrm{mg} \\
(N=26)\end{array}$} & \multirow[t]{2}{*}{$P$ value } & \multicolumn{2}{|c|}{$\begin{array}{l}\text { Pegteograstim } 6.0 \mathrm{mg} \\
(N=57)\end{array}$} & \multicolumn{2}{|c|}{$\begin{array}{l}\text { Pegfilgrastim } 6.0 \mathrm{mg} \\
(N=59)\end{array}$} & \multirow[t]{2}{*}{$P$ value } \\
\hline & No & $\%$ & No & $\%$ & & No & $\%$ & No & $\%$ & \\
\hline Age & & & & & 0.24 & & & & & 0.66 \\
\hline Median & 47.5 & & 47 & & & 49 & & 49 & & \\
\hline Range & $31-73$ & & $29-72$ & & & $32-74$ & & $28-72$ & & \\
\hline Previous chemotherapy & & & & & 0.15 & & & & & 0.62 \\
\hline Yes & 12 & 35 & 6 & 23 & & 6 & 10.5 & 8 & 13.6 & \\
\hline No & 22 & 65 & 20 & 77 & & 51 & 89.5 & 51 & 86.4 & \\
\hline Previous radiotherapy & & & & & 0.48 & & & & & 0.49 \\
\hline Yes & 8 & 23.5 & 6 & 23.1 & & 3 & 5.3 & 6 & 10.2 & \\
\hline No & 26 & 76.5 & 20 & 76.9 & & 54 & 94.7 & 53 & 89.8 & \\
\hline ECOG & & & & & 0.18 & & & & & 0.26 \\
\hline 0 & 22 & 64.7 & 12 & 46.2 & & 33 & 57.9 & 38 & 64.4 & \\
\hline 1 & 11 & 32.4 & 13 & 50.0 & & 24 & 42.1 & 19 & 32.2 & \\
\hline 2 & 1 & 2.9 & 1 & 3.8 & & 0 & 0.0 & 2 & 3.4 & \\
\hline Chemotherapy regimen & & & & & 0.45 & & & & & 0.87 \\
\hline DA & 23 & 67.6 & 18 & 69.2 & & 25 & 43.9 & 25 & 42.4 & \\
\hline TAC & 11 & 32.4 & 8 & 30.8 & & 32 & 56.1 & 34 & 57.6 & \\
\hline Aim of chemotherapy & & & & & 0.33 & & & & & 0.73 \\
\hline Adjuvant/neoadjuvant & 21 & 61.8 & 18 & 69.2 & & 45 & 78.9 & 45 & 76.3 & \\
\hline Palliative & 13 & 39.2 & 8 & 30.8 & & 12 & 21.1 & 14 & 23.7 & \\
\hline
\end{tabular}


Fig. 2 Disposition of patients in the trial

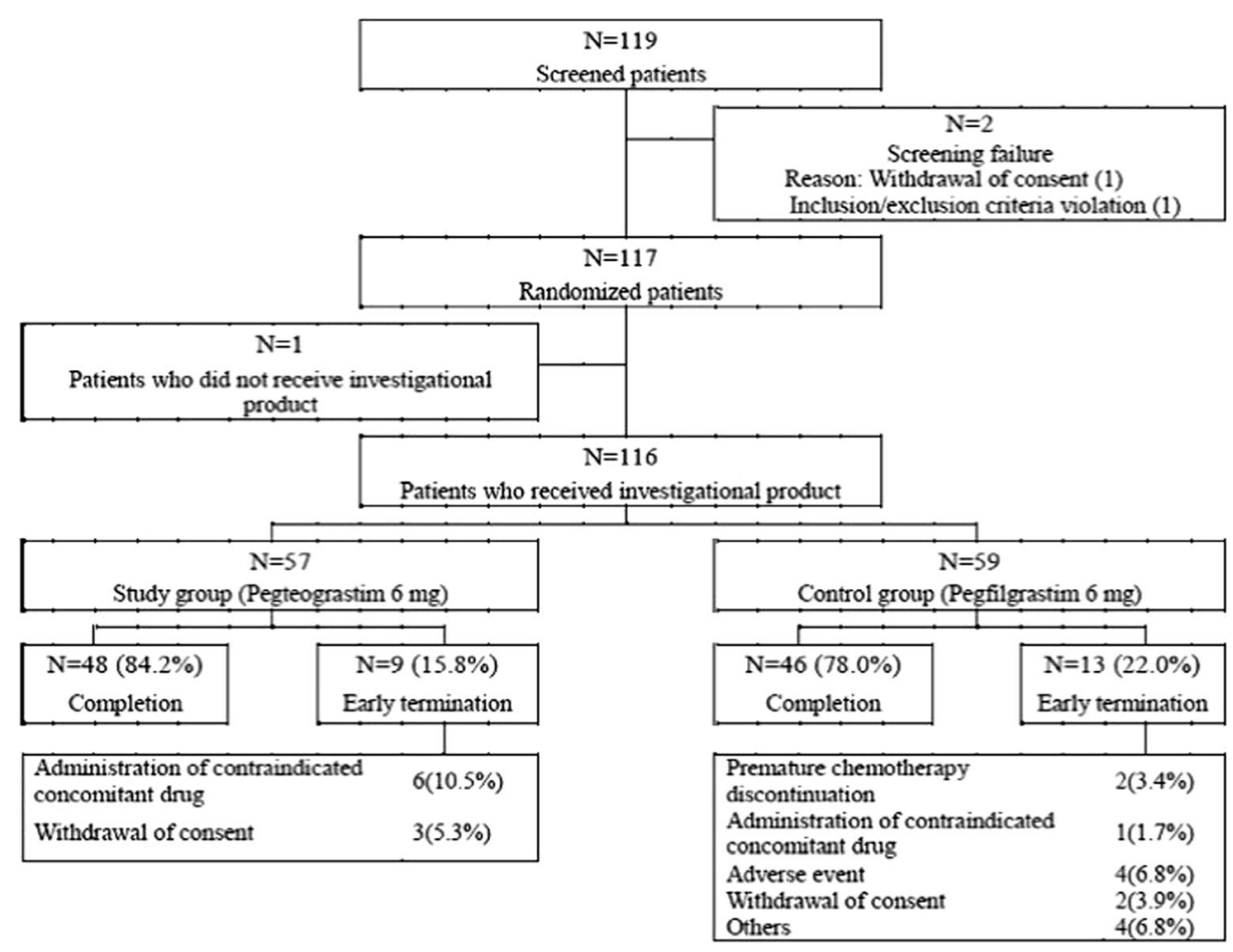

than those of adjuvant/neoadjuvant for both studies, but not significantly so. In both study parts, two groups also had balanced chemotherapy regimens and disease status at the baseline. Twenty-seven of thirty-four patients $(79.4 \%)$ in the $3.6 \mathrm{mg}$ group and $23 / 26$ patients $(88.5 \%)$ in the $6.0 \mathrm{mg}$ group completed all the six chemotherapy cycles in the phase II part. Forty-seven of fifty-eight patients $(81.0 \%)$ in the study group and $46 / 59$ patients $(78.0 \%)$ in the control group completed all the six chemotherapy cycles in the phase III part.

\section{Phase II}

There was no significant difference in the mean duration of grade 4 neutropenia $( \pm \mathrm{SD})$ between group I $(3.6 \mathrm{mg})$ and group II $(6.0 \mathrm{mg}): 1.97 \pm 1.79$ days and $1.54 \pm 0.95$ days, respectively $(p=0.33)$. The median was 2 days in both groups. The times to recovery to ANC $\geq 2000 / \mu \mathrm{L}$ after the ANC nadir were $10.33 \pm 2.68$ days in the $3.6 \mathrm{mg}$ group and $9.69 \pm 1.87$ days in the $6.0 \mathrm{mg}$ group $(p=0.16)$. The incidence rates of $\mathrm{FN}$ in each group were 3 and 2, respectively (9.1 vs. $7.7 \%, p=0.74)$, and the ANC nadir values $(258.2 / \mu \mathrm{L}$ and $213.6 / \mu \mathrm{L}, p=0.13$ ) also showed no significant difference between the two groups (Table 2). The common adverse events (AEs) were nausea, myalgia, and peripheral neuropathy. Most of these AEs had mild to moderate severity and were considered related to the chemotherapy, and there was no difference in the incidence of AEs between the two groups (data not shown). The development of an anti-drug antibody was not observed in both groups. Although there was no significant difference in the primary and secondary endpoints, the Steering Committee selected $6.0 \mathrm{mg}$ as the recommended dose for proceeding to phase III because there was no dosedependent increase in the AEs and the high dose $(6.0 \mathrm{mg})$ was deemed safely administered.

\section{Phase III}

\section{Efficacy}

In the ITT population, the mean $( \pm \mathrm{SD})$ duration of grade 4 neutropenia was comparable in both treatment groups: $1.80 \pm 1.05$ days in the pegfilgrastim group and $1.64 \pm 1.18$ days in the pegteograstim group. The $97.5 \%$ confidence interval for the mean difference was $(-0.57$ and 0.26$)$. As a result, the study met the non-inferiority criterion. Among the secondary efficacy endpoints, the time (in days) to recovery to ANC $\geq 2000 / \mu \mathrm{L}$ in the pegteograstim group was $8.85 \pm 1.45$ days, which was significantly shorter than the $9.83 \pm 1.20$ days in the pegfilgrastim group ( $p<0.0001$ ) (Fig. 3). The incidence of FN did not differ between the two groups. Eleven subjects (19.6\%) in the pegteograstim group and nine $(15.3 \%)$ in the pegfilgrastim group developed FN $(p=0.53)$. Five patients in the pegteograstim group and three in the pegfilgrastim group were hospitalized due to $\mathrm{FN}(p=0.49)$, and 11 patients in the pegteograstim group and nine in the pegfilgrastim group underwent antibiotic therapy $(p=0.53)$.

With regard to the other secondary endpoints, the numeric differences favored pegteograstim over pegfilgrastim, 
Table 2 Efficacy evaluation in phase II and phase III

\begin{tabular}{|c|c|c|c|c|c|c|}
\hline & \multicolumn{2}{|l|}{ Phase II } & \multirow[b]{2}{*}{$\begin{array}{l}P \\
\text { value }\end{array}$} & \multicolumn{2}{|l|}{ Phase III } & \multirow[b]{2}{*}{$P$ value } \\
\hline & $\begin{array}{l}\text { Pegteograstim } \\
3.6 \mathrm{mg} \\
(N=33)\end{array}$ & $\begin{array}{l}\text { Pegteograstim } \\
6.0 \mathrm{mg} \\
(N=26)\end{array}$ & & $\begin{array}{l}\text { Pegteograstim } \\
6.0 \mathrm{mg} \\
(N=56)\end{array}$ & $\begin{array}{l}\text { Pegfilgrastim } \\
6.0 \mathrm{mg} \\
(N=59)\end{array}$ & \\
\hline Duration (days) of grade 4 neutropenia & $1.97 \pm 1.79$ & $1.54 \pm 0.95$ & 0.33 & $1.64 \pm 1.18$ & $1.80 \pm 1.05$ & $\begin{array}{c}-0.15 \pm 1.11^{\mathrm{a}} \\
{[-0.57,0.26]}\end{array}$ \\
\hline ANC recovery time (days) ${ }^{a}$ & $10.33 \pm 2.68$ & $9.69 \pm 1.87$ & 0.16 & $8.85 \pm 1.45$ & $9.83 \pm 1.20$ & $<0.0001$ \\
\hline Incidence of FN $(N, \%)^{\mathrm{b}}$ & $3(9.1)$ & $2(7.7)$ & 0.74 & $11(19.6)$ & $9(15.3)$ & 0.53 \\
\hline ANC nadir ${ }^{\mathrm{c}}$ & $258.2 \pm 249.9$ & $213.6 \pm 240.4$ & 0.13 & $290.4 \pm 362.0$ & $198.3 \pm 199.7$ & 0.09 \\
\hline ANC on day $7^{d}$ & NA & NA & NA & $1064.1 \pm 1098.7$ & $918.8 \pm 869.3$ & 0.32 \\
\hline $\begin{array}{l}\text { Incidence of persistent grade } 4 \text { neutropenia over } \\
3 \text { days or longer }\end{array}$ & NA & NA & NA & $8(14.6)$ & $13(22.4)$ & 0.28 \\
\hline $\begin{array}{l}\text { Incidence of delay or dose reduction in } \\
\text { chemotherapy }{ }^{f}\end{array}$ & NA & NA & NA & $12(23.1)$ & $22(37.9)$ & 0.09 \\
\hline
\end{tabular}

$N A$ not available

${ }^{\text {a }}$ Time (days) to recovery to $\mathrm{ANC} \geq 2000 / \mathrm{mm}^{3}$ following ANC nadir during cycle 1

${ }^{\mathrm{b}}$ Frequency and percentage of patient with at least one febrile neutropenia during each cycle

${ }^{\mathrm{c}}$ ANC nadir during cycle 1

${ }^{\mathrm{d}}$ Mean ANC value on day 7 at each cycle

${ }^{\mathrm{e}}$ Frequency and percentage of patient with persistent grade 4 neutropenia over 3 days or longer during cycle 1

${ }^{\mathrm{f}}$ Frequency and percentage of patient with at least one delay or dose reduction in chemotherapy due to neutropenia at each cycle

although they were not statistically significant. The incidences of persistent grade 4 neutropenia over 3 days were 8 (14.6\%) in the pegteograstim group and $13(22.4 \%)$ in the pegfilgrastim group $(p=0.28)$. The numbers of cases in which a delay or dose reduction in chemotherapy was required were $12(23.1 \%)$ and $22(37.9 \%)$ in the pegteograstim and pegfilgrastim groups, respectively $(p=0.09)$. The depths of neutropenia (the ANC nadirs) in cycle 1 were $290.4 \pm 362.0$ / $\mu \mathrm{L}$ in the pegteograstim group and $198.3 \pm 199.7 / \mu \mathrm{L}$ in the pegfilgrastim group $(p=0.09)$. The ANC counts on day 7 of cycle 1 were $1064.1 \pm 1098.7$ and $918.8 \pm 869.3(p=0.32)$.
The subgroup analyses according to the type of chemotherapy (DA vs. TAC). In the chemotherapy with $6.0 \mathrm{mg}$ pegteograstim, there was no significant difference in the mean duration of grade 4 neutropenia $( \pm \mathrm{SD})$ between the TAC regimen and the DA regimen: $1.84 \pm 1.21$ days and $1.4 \pm 1.15$ days, respectively $(p=0.26)$. In the chemotherapy with $6.0 \mathrm{mg}$ pegfilgrastim, there was no significant difference in the mean duration of grade 4 neutropenia $( \pm \mathrm{SD})$ between the TAC regimen and the DA regimen: $1.96 \pm 0.93$ days and $1.68 \pm 1.12$ days, respectively $(p=0.31)$.
Fig. 3 Changes in mean absolute neutrophil count (ANC) chemotherapy cycle 1 (phase III part)

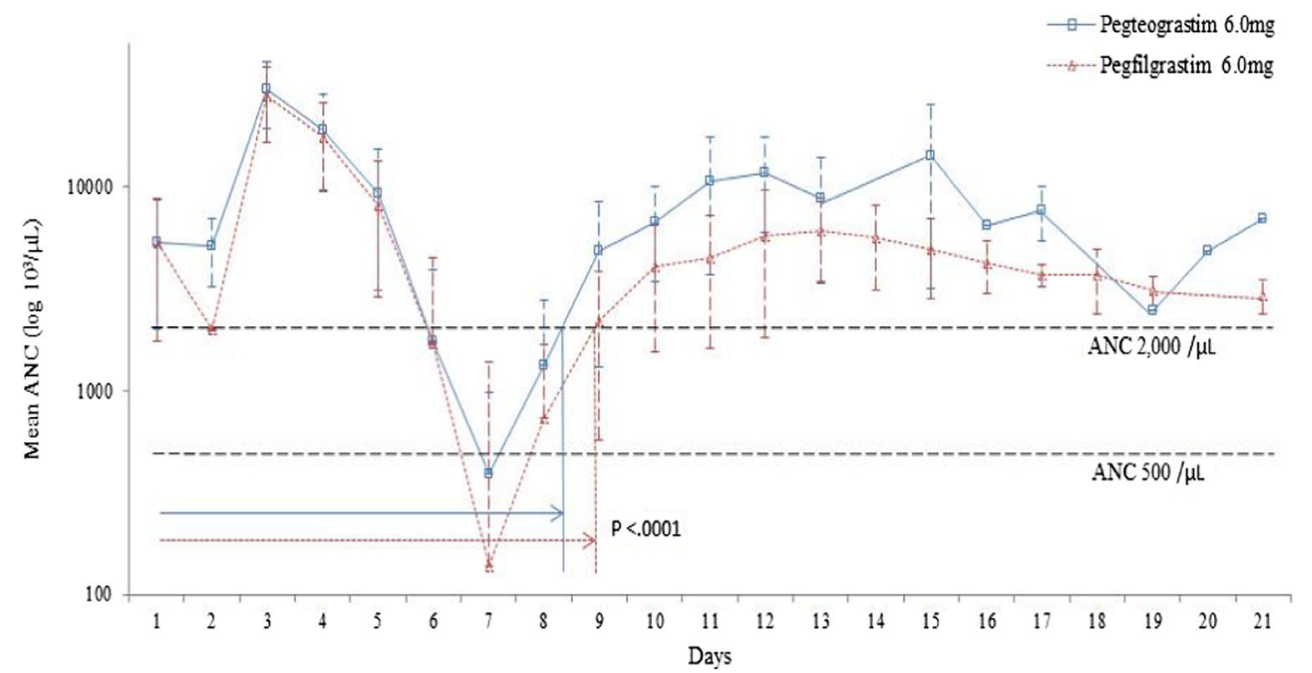


Also, the history of chemotherapy or radiotherapy, or the treatment setting (adjuvant/neoadjuvant vs. palliative) showed no significant difference in the primary or secondary outcomes between the pegteograstim and pegfilgrastim groups (data not shown).

\section{Safety}

AEs occurred in 55 patients $(96.5 \%)$ in the pegteograstim group and in 59 patients $(100 \%)$ in the pegfilgrastim group. The frequent AEs were nausea, myalgia, constipation, peripheral neuropathy, and decreased appetite, most of which were due to the chemotherapy, especially the docetaxel chemotherapy (Table 3). Adverse drug reactions (ADRs), wherein the causal relationship between the AEs and the study drugs could not be excluded, were 5.3 and $3.4 \%$ in the pegteograstim group and the pegfilgrastim group, respectively. The ADRs were leukocytosis, blurred vision, injection site pain, pyrexia, dizziness, headache, and peripheral sensory neuropathy. One out of 116 patients showed leukocytosis $(\geq 30,000 / \mu \mathrm{L})$. The marked leukocytosis $(75,000 / \mu \mathrm{L})$ was observed from day 2 of cycle 5 to day 1 of cycle 6 in the pegteograstim group and was resolved without any complication. There was no significant difference in the incidence of AEs or ADRs between the groups.

\section{Discussion}

The results of this phase II/III study demonstrated the noninferiority of pegteograstim to pegfilgrastim in the prevention of severe neutropenia after high-risk chemotherapy in breast cancer patients. The duration of grade 4 neutropenia and the incidence of FN in the patients who received prophylactic pegteograstim were comparable to those in the patients who received pegfilgrastim. The duration of grade 4 neutropenia observed in phase III (1.64 days) substantiates the result in phase II of 1.54 days for the $6.0 \mathrm{mg}$ group. The median duration of grade 4 neutropenia in the patients who underwent docetaxel plus doxorubicin chemotherapy but were not treated with G-CSF has been reported to range from 4 to 7 days [11-13]. Based on the results of this study, both pegteograstim and pegfilgrastim are expected to reduce the duration of grade 4 neutropenia by more than half. Primary prophylaxis with CSF is known to reduce the risks of hospitalization and allcause mortality in breast cancer patients undergoing chemotherapy [14]. Therefore, pegteograstim is expected to have a clinically relevant effect on the prevention of FN and on the safety of breast cancer patients undergoing myelosuppressive chemotherapy.

In the phase II part of this study, there was no significant difference between the 3.6 and $6.0 \mathrm{mg}$ pegteograstim groups in terms of the duration of grade 4 neutropenia and the other secondary endpoints. However, in the previously reported phase I study of pegteograstim in healthy subjects, the higher doses $(100$ and $300 \mu \mathrm{g} / \mathrm{kg})$ showed higher-level activity in terms of the maximum ANC and $\mathrm{CD} 34^{+}$cell counts as well as superior pharmacokinetic parameters [10]. In addition, no dose-dependent increase in AEs or ADRs was observed in phase II of this study. Therefore, the high dose $(6.0 \mathrm{mg})$ tested in this study was considered within the maximum dose range for safe use and was selected as the dose for the study group in phase III.

Among the secondary efficacy endpoints, the time to recovery to $\mathrm{ANC} \geq 2000 / \mu \mathrm{L}$ in the pegteograstim group was significantly shortened by 1 day compared with that in the pegfilgrastim group ( 8.85 vs. 9.83 days, $p<0.0001$ ). In the previously reported phase I study, a more significantly increased activity of pegteograstim on $\mathrm{ANC}$ and $\mathrm{CD} 34^{+}$cells than with the same dose of pegfilgrastim was suggested [10]. The study showed that although pegteograstim has $19 \%$ lower systemic exposure than pegfilgrastim at the same dose, it is associated with a $20 \%$ higher area under the effect-time curve (AUEC) values for the plasma $\mathrm{ANC}$ and $\mathrm{CD} 34^{+}$cell counts. The lower systemic exposure with the higher activity of pegteograstim is explained by its more prominent target-mediated drug disposition (TMDD) pattern of elimination than that of pegfilgrastim. Much of pegteograstim is eliminated by receptors on neutrophil or $\mathrm{CD} 34^{+}$cells, and increased clearance indicates increased activity on ANC and $\mathrm{CD} 34^{+}$cells (i.e., increased receptors).

Although there was no difference between the groups, the incidences of FN in this study $(19.6 \%$ in the pegteograstim group and $15.3 \%$ in the pegfilgrastim group) were somewhat higher than those in other studies that reported the incidence of FN as less than $10 \%$ using prophylactic G-CSF [15-19]. However, since the incidences of FN greatly vary according to the differences in the definition of FN, the chemotherapy regimens and duration of treatment, the primary prophylaxis with antibiotics, and the eligibility for prior chemotherapies, the inter-trial comparison should be conducted with care. In this study, the definition of $\mathrm{FN}$ is less restrictive $\left(\geq 38.3{ }^{\circ} \mathrm{C}\right.$ measured once or two or more instances of $\geq 38.0^{\circ} \mathrm{C}$ each taken within 1-24 $\mathrm{h}$ after the initial measurement), and more than $10 \%$ of the patients had chemotherapy treatment before their inclusion in this study.

The limitations of this study were the small number of patients and the defined treatment regimen in the study population. However, similar to pegfilgrastim, pegteograstim showed a favorable safety profile. Its frequent AEs were myalgia, peripheral neuropathy, decreased appetite, nausea, and constipation, most of which are known to be associated with chemotherapy. Based on this study, further clinical trials are being conducted and planned. A post-marketing surveillance study is now being performed to assess the further safety profile of pegteograstim, and additional trials with various cancer types, patient populations, and treatment regimens are warranted. 
Table 3 Safety evaluation (Adverse events)

\begin{tabular}{|c|c|c|c|c|c|c|c|c|c|c|}
\hline & \multicolumn{5}{|l|}{ Phase II } & \multicolumn{5}{|l|}{ Phase III } \\
\hline & \multicolumn{2}{|c|}{$\begin{array}{l}\text { Pegteograstim } 3.6 \mathrm{mg} \\
(N=34)\end{array}$} & \multicolumn{2}{|c|}{$\begin{array}{l}\text { Pegteograstim } 6.0 \mathrm{mg} \\
(N=26)\end{array}$} & \multirow[t]{2}{*}{$P$ value } & \multicolumn{2}{|c|}{$\begin{array}{l}\text { Pegteograstim } 6.0 \mathrm{mg} \\
(N=57)\end{array}$} & \multicolumn{2}{|c|}{$\begin{array}{l}\text { Pegfilgrastim } 6.0 \mathrm{mg} \\
(N=59)\end{array}$} & \multirow[t]{2}{*}{$P$ value } \\
\hline & All grade & Grade $3 / 4$ & All grade & Grade 3/4 & & All grade & Grade $3 / 4$ & All grade & Grade $3 / 4$ & \\
\hline Total $(N, \%)$ & $34(100)$ & $6(17.6)$ & $26(100)$ & $2(7.7)$ & 0.45 & $55(96.5)$ & $16(28.1)$ & $59(100)$ & $14(23.7)$ & 0.59 \\
\hline Anemia & $2(5.9)$ & - & - & - & - & $5(8.8)$ & $1(1.8)$ & 7 (11.9) & $2(3.4)$ & 1.00 \\
\hline Febrile neutropenia & $4(11.8)$ & $4(11.8)$ & $2(7.7)$ & $1(3.8)$ & 0.38 & $11(19.3)$ & $11(19.3)$ & $9(15.3)$ & $9(15.3)$ & 0.56 \\
\hline Decreased appetite & $6(17.6)$ & - & $6(23.1)$ & - & - & $20(35.1)$ & $1(1.8)$ & $26(44.1)$ & $1(1.7)$ & 1.00 \\
\hline Nausea & $19(55.9)$ & - & $17(65.4)$ & - & - & $35(61.4)$ & $2(3.5)$ & $39(66.1)$ & $1(1.7)$ & 0.62 \\
\hline Vomiting & $6(17.6)$ & - & $3(11.5)$ & - & - & $16(28.1)$ & $1(1.8)$ & $8(13.6)$ & - & 0.49 \\
\hline Stomatitis & $3(8.8)$ & - & $2(7.7)$ & - & - & $8(14.0)$ & - & $6(10.2)$ & - & - \\
\hline Diarrhea & $6(17.6)$ & - & $7(26.9)$ & - & - & $20(35.1)$ & $3(5.3)$ & $17(28.8)$ & $2(3.4)$ & 0.68 \\
\hline Constipation & $10(29.4)$ & - & $6(23.1)$ & - & - & $24(42.1)$ & - & $27(45.8)$ & - & - \\
\hline Asthenia & $5(14.7)$ & - & $1(3.8)$ & - & - & $11(19.3)$ & $1(1.8)$ & $13(22.0)$ & - & 0.49 \\
\hline Generalized edema & $11(32.4)$ & - & $5(19.2)$ & - & - & $6(10.5)$ & - & $8(13.6)$ & - & - \\
\hline Myalgia & $19(55.9)$ & $2(5.9)$ & $11(42.3)$ & - & 0.50 & $32(56.1)$ & $2(3.5)$ & $35(59.3)$ & $4(6.8)$ & 0.68 \\
\hline Headache & $5(14.7)$ & - & $4(15.4)$ & - & - & $13(22.8)$ & $2(3.5)$ & $15(25.4)$ & - & 0.24 \\
\hline Peripheral neuropathy & $6(17.6)$ & - & $8(30.8)$ & $1(3.8)$ & 0.43 & $15(26.3)$ & & $13(22.0)$ & - & - \\
\hline
\end{tabular}

\section{Conclusion}

This study showed that $6.0 \mathrm{mg}$ pegteograstim is as effective as $6.0 \mathrm{mg}$ pegfilgrastim in reducing the risk of chemotherapyinduced neutropenia with a tolerable safety profile.

Acknowledgments This study was sponsored by Green Cross Corporation. The authors thank Korean Cancer Study Group, the study nurses, and the patients for participating in this trial. Author Do-Youn Oh and author Seock-Ah Im are jointly responsible for the submission.

Conflicts of interest The authors declare that they have no competing interests.

Open Access This article is distributed under the terms of the Creative Commons Attribution-NonCommercial 4.0 International License (http://creativecommons.org/licenses/by-nc/4.0/), which permits any noncommercial use, distribution, and reproduction in any medium, provided you give appropriate credit to the original author(s) and the source, provide a link to the Creative Commons license, and indicate if changes were made.

\section{References}

1. National Comprehensive Cancer Network. Myeloid growth factors (version 2. 2014).https://www.nccn.org/store/login/login.aspx? ReturnURL=http://www.nccn.org/professionals/physician_gls/pdf/ myeloid_growth.pdf. Accessed February 19, 2014.

2. Morstyn G, Campbell L, Souza LM, Alton NK, Keech J, Green M, et al. (1988) Effect of the granulocyte-colony-stimulating factor on neutropenia induced by cytotoxic chemotherapy. Lancet 331(8587):667-672
3. Johnston E, Crawford J, Blackwell S, et al. (2000) Randomized dose escalation study of SD/01 compared with daily filgrastim in patients receiving chemotherapy. J Clin Oncol 18(13):2522-2528

4. Molineux G, Kinstler O, Briddell B, et al. (1999) A new form of filgrastim with sustained duration in vivo and enhanced ability to mobilize PBPC in both mice and humans. Exp Hematol 27:1724 1734

5. Hlmes FA, O'Shaughnessy JA, Vukelja S, Jones SE, Shogan J, Savin M, et al. (2002) Blinded, randomized, multicenter study to evaluate single-administration pegfilgrastim once per cycle versus daily filgrastim as an adjunct to chemotherapy in patients with highrisk stage II or stage III/IV breast cancer. J Clin Oncol 20(3):727731

6. Green MD, Koelbl H, Baselga J, Galid A, Guillem V, Gascon P, et al. (2003). International Pegfilgrastim 749 Study Group. A randomized double-blind multicenter phase III study of fixed-dose single-administration pegfilgrastim versus daily filgrastim in patients receiving myelosuppressive chemotherapy. Ann Oncol (1): 29-35.

7. Naeim A, Henk HJ, Becker L, Chia V, Badre S, Li X, et al. (2013) Pegfilgrastim prophylaxis is associated with a lower risk of hospitalization of cancer patients than filgrastim prophylaxis: a retrospective United States claims analysis of granulocyte colonystimulating factors (G-CSF). BMC Cancer 13:11

8. Lyman GH, Lalla A, Barron RL, Dubois RW (2009) Costeffectiveness of pegfilgrastim versus filgrastim primary prophylaxis in women with early-stage breast cancer receiving chemotherapy in the United States. Clin Ther 31(5):1092-1104

9. Lyman G, Lalla A, Barron R, Dubois RW (2009) Costeffectiveness of pegfilgrastim versus 6-day filgrastim primary prophylaxis in patients with non-Hodgkin's lymphoma receiving CHOP-21 in United States. Curr Med Res Opin 25(2):401-411

10. Shin KH, Lim KS, Lee H, Jang IJ, Yu KS (2014) An assessment of the pharmacokinetics, pharmacodynamics, and tolerability of GCPGC, a novel pegylated granulocyte-colony-stimulating factor (G-CSF), in healthy subjects. Investig New Drugs 32(4):636-643 
11. Misset JL, Dieras V, Gruia G, Bourgeois H, Cvitkovic E, Kalla S, et al. (1999) Dose-finding study of docetaxel and doxorubicin in the first-line treatment of patients with metastatic breast cancer. Ann Oncol 10(5):553-560

12. Nabholtz JM, Falkson C, Campos D, Szanto J, Martin M, Chan S, et al. (2003) TAX 306 Study Group. Docetaxel and doxorubicin compared with doxorubicin and cyclophosphamide as first-line chemotherapy for metastatic breast cancer: results of a randomized, multicenter, phase III trial. J Clin Oncol. Mar. 15; 21 (6): 968-75. Erratum in:. J Clin Oncol 21(10):2048

13. del Giglio A, Eniu A, Ganea-Motan D, Topuzov E, Lubenau H (2008) $\mathrm{XM} 02$ is superior to a placebo and equivalent to Neupogen in reducing the duration of severe neutropenia and the incidence of febrile neutropenia in cycle 1 in breast cancer patients receiving docetaxel/ doxorubicin chemotherapy. BMC Cancer 8:332

14. Renner P, Milazzo S, Liu JP, Zwahlen M, Birkmann J, Horneber M (2012) Primary prophylactic colony-stimulating factors for the prevention of chemotherapy-induced febrile neutropenia in breast cancer patients. Cochrane Database Syst Rev 10:CD007913

15. Kosaka Y, Rai Y, Masuda N, Takano T, Saeki T, Nakamura S, et al. (2015) Phase III placebo-controlled, double-blind, randomized trial of pegfilgrastim to reduce the risk of febrile neutropenia in breast cancer patients receiving docetaxel/cyclophosphamide chemotherapy. Support Care Cancer 4:1137-1143
16. Bondarenko I, Gladkov OA, Elsaesser R, Buchner A, Bias P (2013) Efficacy and safety of lipegfilgrastim versus pegfilgrastim: a randomized, multicenter, active-control phase 3 trial in patients with breast cancer receiving doxorubicin/docetaxel chemotherapy. BMC Cancer 13:386

17. Martín M, Lluch A, Seguí MA, Ruiz A, Ramos M, Adrover E, et al. (2006) Toxicity- and health-related quality of life of breast cancer patients receiving adjuvant docetaxel, doxorubicin, and cyclophosphamide (TAC) or 5-fluorouracil, doxorubicin, and cyclophosphamide (FAC): impact of adding a primary prophylactic granulocytecolony-stimulating factor to the TAC regimen. Ann Oncol 17(8): 1205-1212

18. Aapro MS et al. (2011) 2010 update of EORTC guidelines for the use of granulocyte colony stimulating factor to reduce the incidence of chemotherapy-induced febrile neutropenia in adult patients with lymphoproliferative disorders and solid tumours. Eur J Cancer 47: $8-32$

19. von Minckwitz G, Kümmel S, du Bois A, et al (2008) Pegfilgrastim \pm ciprofloxacin for primary prophylaxis with TAC (docetaxel/doxorubicin/cyclophosphamide) chemotherapy for breast cancer. Results from the GEPARTRIO Study Annals of Oncology 19: 292-298 